\title{
A review of Network Topology
}

\section{Ruojing Jiang}

School of Mathematical Sciences, Fudan University, Shanghai 200433, China.

Ruojing_Jiang@126.com

Keywords: Bus topology, Ring topology, Star topology, Tree topology, Mesh Topology, Hierarchical Topology.

\begin{abstract}
A network topology is how computers, printers, and other devices are connected over a network. It describes the layout of wires, devices, and routing paths. Essentially there are six different common topologies you should familiarize yourself with: Bus, Ring, Star, Extended Star, Hierarchical, and Mesh. In this paper, the recent development of six different common topologies are summarized.
\end{abstract}

\section{Introduction}

In communication networks, a topology is a usually schematic description of the arrangement of a network, including its nodes and connecting lines. There are two ways of defining network geometry: the physical topology and the logical (or signal) topology.

Network topology is the layout of the connections of a computer network. Network topologies may be physical or logical. Physical topology means the physical design of a network including the locations, devices and cables.

There are six basic topologies: Bus topology, Star topology, Ring topology, Tree topology, Mesh topology, and Hierarchical topology. Which of these is chosen rest with what devices need to be connected, how reliable it has to be, and the cost associated with cabling.

In this paper, a review of the recent development of the above six different common topologies are provided.

\section{The development of six different common topologies}

Star Topology. A star topology is designed with each node connected directly to a central network hub, switch, or concentrator, shown in Figure 1.

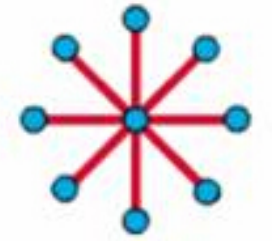

Fig.1 Star topology

Before continuing transmit to its destination, data on a star network passes through the switch, hub or concentrator. All functions of the network is managed and controlled by the hub, switch or concentrator. Three star-shaped molecules were designed and synthesized by Nadzeya A. Kukhta et al. [1]. The obtained compounds are characterized by UV and fluorescence spectroscopies, thermogravimetric analysis, cyclic voltammetry, differential scanning calorimetry, and time-of-flight and CELIV techniques. In metropolitan network with different topologies which uses the link bandwidth efficiently and increases the capacity of the system [1]. Star-ring architecture with the cascade add/drop transceiver structure is proposed by Wen-Piao Lin et al. [1]. This new structure is used for subcarrier multiplexed PON with 118 optical network units (ONUs) at $2.2 \mathrm{~km}$. The system consists of many concatenated ring subnets on the lower level with self-healing capabilities and a star network on the upper level. 
Ring Topology. In the ring network topology shown in Figure 2, the workstations are connected in a closed loop configuration. Adjacent pairs of workstations are directly connected. Other pairs of workstations are indirectly connected, the data passing through one or more intermediate nodes.

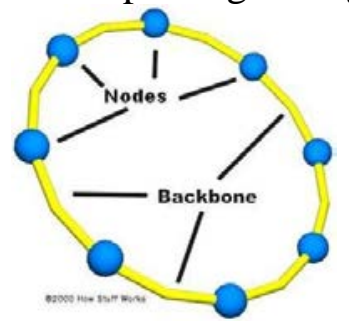

Fig.2 Ring topology

It is observed that the formal proposed architecture were limited to lesser data rates and investigated at lesser transmission distance [2]. And no hybrid network with maximum number of users is proposed till now. AWDM network using ring-tree-star hybrid topology is first proposed by Simranjit Singh et al. [2]. In their study, the performance of proposed WDM network ring-tree-star hybrid topology is investigated with eight OADM nodes. Eight optical add/drop multiplexers are used to make ring structure; four channels are used to transmit data with 10 Gbps data rate [2].

Bus Topology. In the bus network topology shown in Figure 3, every workstation is connected to a main cable called the bus. Therefore, in effect, each workstation is directly connected to every other workstation in the Network.

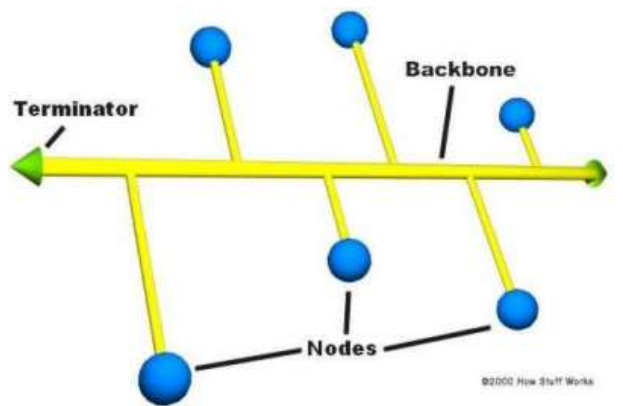

Fig.3 Bus topology

In the last decade, several MAC protocols were proposed for MAN [3]. Among of these protocals, Most are using either the bus or the ring topology, and the most important of the bus topologies is the Dual Bus protocol [3]. Although the DSDR protocol has excellent utilization, it does not possess reservation mechanism. As a result, the access delay may be quite high in this protocol. In order to avoid such disadvantages, a new End-Connected dual bus topology and a modified MAC protocol to increase the utilization of the dual bus protocol are proposed and developed by M.R. Ramapriya et al. [4]. Token passing scheme is used as the design base of the proposed protocols. The destination stripping mechanism is included and a provision is made to transmit on the additional free slots which is available through connected ends [4].

Tree Topology. The tree network topology, shown in Figure 4, uses two or more star networks connected together. The central computers of the star networks are connected to a main bus. Thus, a tree network is a bus network of star networks.

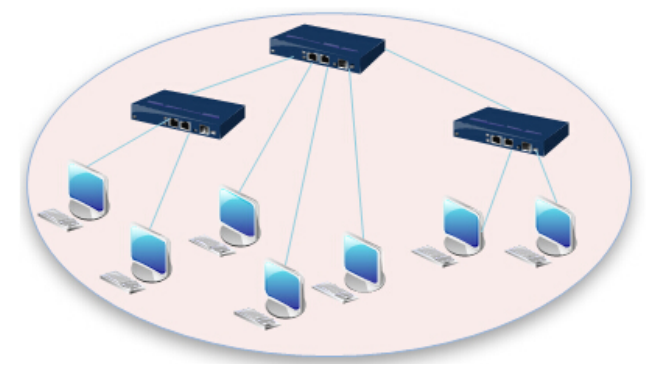

Fig.4 The tree network topology 
Building a tree topology over a network is a well-known technique where the information the tree adds over the system can be really useful for many different applications like routing, search, etc. a tree based topology in the network is first applied for the broadcasting process by Patricia Ruiz et al.[5]. The feasibility of relying on a tree-based topology management structure for mobile ad hoc networks and in particular for VANETs is assessed. Research results show it is feasible to apply this topology over a vehicular network [5].

Mesh Topology. The mesh network topology, shown in Figure 5, employs either of two schemes, called full mesh and partial mesh. In the full mesh topology, each workstation is connected directly to each of the others. In the partial mesh topology, some workstations are connected to all the others, and some are connected only to those other nodes with which they exchange the most data.

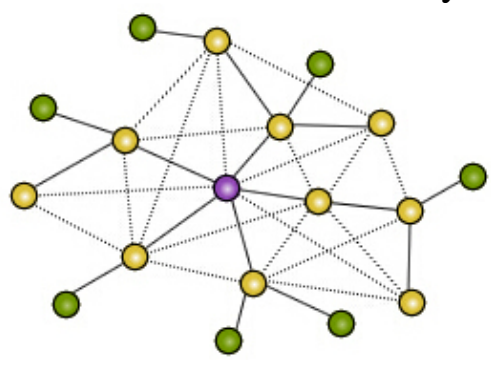

Fig.5 Mesh Topology

In order to facilitate local traffic, some photonic NoC architectures are designed to utilize electrical interconnect for fast local switching, an optical NoC with global crossbar topology is proposed Batten et al. [6]. Where DRAM and processing cores are connected with a hybrid opto-electrical global optical crossbar and divided in sub-mesh and [6]. A clustered optical interconnection network Corona with broadcasting support is proposed by Vantrease et al.[6]. Two novel optical architectures for global communication is proposed by Pan et al.[6], which includes Firefly and a further improved architecture Flexishare. The proposed topology reduces the overall insertion loss of wave guide crossings.

Hierarchical Topology. The Hierarchical Topology, shown in Figure 6, is much like the Star Topology, except that it doesn't use a central node. Although Cisco prefers to call this Hierarchical, you may see it as instead referred to as the Tree Topology. This type of topology suffers from the same centralization flaw as the Star Topology. If the device that is on top of the chain fails, consider the entire network down. Obviously this is impractical and not used a great deal in real applications.

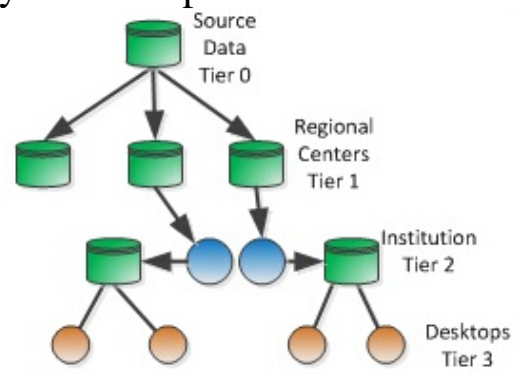

Fig.6 Hierarchical Topology

A combination of any two or more network topologies. Note 1: Instances can occur where two basic network topologies, when connected together, can still retain the basic network character, and therefore not be a hybrid network. For example, a tree network connected to a tree network is still a tree network. Therefore, a hybrid network accrues only when two basic networks are connected and the resulting network topology fails to meet one of the basic topology definitions. For example, two star networks connected together exhibit hybrid network topologies. Note 2: A hybrid topology always accrues when two different basic network topologies are connected. 


\section{Conclusion}

This paper provides a review of the recent development of main six different common topologies: Bus, Ring, Star, Extended Star, Hierarchical, and Mesh. Each one has advantages and disadvantages, for example, advantages of a star topology are: easy to install and wire, no disruptions to the network when connecting or removing devices, easy to detect faults and to remove parts, while the disadvantages are: requires more cable length than a linear topology, if the hub, switch, or concentrator fails, nodes attached are disabled, more expensive than linear bus topologies because of the cost of the hubs, etc.. In summary, in order to promote the development of network topology, further research should focus on how to avoid its disadvantages.

\section{References}

[1] Nadzeya A. Kukhtaa, Jurate Simokaitiene, Dmytro Volyniuk, Jolita Ostrauskaite,Juozas V. Grazulevicius, Gytis Juska, Vygintas Jankauskas. Effect of linking topology on the properties of star-shaped derivatives of triazine and fluorene. Synthetic Metals, Volume 195, September 2014, Pages 266-275.

[2]Simranjit Singh, Raman, R.S. Kaler. Investigation of wavelength division multiplexed hybrid ring-tree-star network topology to enhance the system capacity. Optik 125 (2014) 6516-6519.

[3] Cheng Zhang, Weiming Ma, Chi Sun.A switchable high-speed fiber-optic ring net topology and its method of high-performance synchronization for large-capacity power electronics system. Electrical Power and Energy Systems 57 (2014) 335-349.

[4] M.R. Ramapriya, S. Beotra, M. Mukherjee, R.V. Raja Kumar, P. Dhar. An end-connected dual bus topology for metropolitan area networks. Computer Networks 31 (1999) 445-456

[5] Patricia Ruiz, Bernabé Dorronsoro, Pascal Bouvry, Lorenzo Tardón. Information dissemination in VANETs based upon a tree topology. Ad Hoc Networks 10 (2012) 111-127.

[6] Kai Feng, Yaoyao Ye, Jiang Xu. A formal study on topology and floorplan characteristics of mesh and torus-based optical networks-on-chip. Microprocessors and Microsystems 37 (2013) 941-952. 